\title{
Marketing Mix and Retail Practices Aimed for Demographics of Customers of Selected Retail Stores
}

\author{
Dr.B. Archana \\ CVR College of Engineering, Department of Management Studies, Ibrahimpatan, R.R.District, A.P., India. \\ Email: archana_boda@yahoo.co.in
}

\begin{abstract}
India is a land of increasing retail opportunities. Retailing has bright prospects, propelled by the changes taking place among the demographics of customers. In this relevance, the retailers marketing strategy takes into consideration, factors like pricing, location, brands and retail practices. The present study attempts to identify the marketing mix and to score the retail practices of selected retail stores offered to the identified set of demographics of customers.
\end{abstract}

Index Terms-Retail stores, Retail practices, Demographics, Marketing Mix.

\section{INTRODUCTION}

Retailing is one of the largest industries in India and one of the biggest sources of employment in the country. Although the retailing industry has existed in our country for centuries, it is only in the recent past that it has witnessed such a tremendous growth. Retailing is derived from the French word 'retailier', which means, "to cut a piece off". Thus, retailing can be defined as a set of business activities that adds value to the products and services sold to the final consumers for their personal, family or household use.

The existence of the customer is integral to the existence of the retail store. The ability to understand customers is the key to developing a successful retail strategy. To be able to satisfy the customers, it is necessary to understand them in terms of their demographics, which include gender, age, race, socioeconomic status and other statistics. As competition increases and the customer becomes more and more knowledgeable and demanding, the retailer needs this knowledge to stay ahead of his competitors and to build a competitive advantage. In this perspective, a retail store takes into consideration, factors like marketing mix which includes pricing, branding \& location of stores and also the various retail practices that it offers to its demographics of the customers. In this backdrop, the present paper attempts to study the following objectives:

\section{OBJECTIVES OF THE STUDY}

1. To identify the demographics of the customers of selected retail stores.

2. To study the marketing mix offered to the identified demographics.

3. To score the retail stores for their retail practices offered to the identified demographics.

\section{THE SOURCES OF DATA}

For the purpose of the study a structured questionnaire was designed and the data was collected from Retailers of various selected retail stores located in the twin cities of Hyderabad and Secunderabad of Andhra Pradesh.

\section{DATA ANALYSIS}

The 100 retailers selected as the sample for the purpose of the study were categorized into twelve store types for analysis as Department Stores, Hyper Markets, Footwear Outlets, Super Markets, Optical Showrooms, Electronic Stores, Food Retailers, Furniture Stores, Gifts and Novelties, Jewellery Stores, Garments Stores and Other Retail stores.

Objective 1: To identify the demographics of the customers of selected retail stores.

\subsection{Gender}

Table 1.1.in Appendix depicts that majority of the selected retail stores were retailing both for male and female customers.

\subsection{Age}

Table 1.2 in Appendix depicts that all the selected retailers considered for the study were retailing for all the age groups of customers.

\subsection{Socio-Economic Status}

Table 1.3 in Appendix depicts that the selected retailers for the purpose of the study had majority of the customers who belonged to the Middle class of SocioEconomic levels. The second majority of the customers belonged to Upper Middle class.

Objective 2: To study the marketing mix offered to the identified demographics

\subsection{Brands Sold}

As the Table 2.1 in Appendix depicts, the selected retailers of the study were on a majority selling national brands for their customers.

The article is based on the $\mathrm{Ph}$. D Thesis of the author. 


\subsection{Pricing Strategies}

The retailers of various selected store types had given their responses with regard to their Pricing Strategies, as shown in the Table 2.2 in Appendix. Majority of them followed MRP pricing strategy, whereas, Odd pricing strategy and EDLP pricing strategy were practiced only by 1 percent of them.

\subsection{Location Criteria}

The retailers of selected store types were asked to give preferences for considering the criteria for locating their retail stores. The Table 2.3 in Appendix it is clear that 100 percent of these retailers on the first basis opined in favor of criteria like Visitors group attraction, Security, Well connected transport, Planned Shopping area, Occupancy costs, Landlord control and Tax incentives. 50 percent of the retailers gave their next preference on location criteria for Competition factors. And the other criteria listed in the table were given the subsequent preferences, by the various retailers.

Objective 3: To score the retail stores for their retail practices offered to the identified demographics

\subsection{Measuring Retail Practices (Weights)}

The 100 retailers considered for the study were asked to state their retail practices, by ranking the various elements as: Usually, Sometimes and Rarely. These 100 retailers were grouped into twelve types based on their store type. Each of these selected store types were given scores for their customer practices followed, by assigning weights for their responses, as ' 5 ' for 'usually', ' 3 ' for 'sometimes' and ' 1 ' for 'rarely'. The same is shown Table 3.1 in Appendix.

\subsection{Scoring the Retail Practices}

From the above retail practices measured for the selected retail stores, the following are the scores assigned as shown in the Table 3.2 in Appendix. As the Mean value of the Department Stores is high among the 12 selected retail stores considered for the study, it could be concluded that the Department stores had ranked maximum score for their various retail practices offered to the customers belonging to the set of demographics.

\section{CONCLUSIONS}

Retailing is a dynamic industry which is constantly changing due to shifts in the needs of the customers and the growth of competitors. The expectations of the customers take the shape of the products, location, offerings and other elements that help the customer choose a retail store and decide on patronizing it. One of the biggest challenges for the retailers today, is to carefully plan clearly and to build a long-term relationship with the customers. Retailers also need to identify the demographics of the customers to target them and understand them. Thus, retailing is a blend of marketing mix and retail practices offered to benefit any set of demographic customers.

\section{BIBILIOGRAPHY}

1. Mc Kenna, Regis, Relationship Marketing: Successful Strategies for the Age of the Customers, Addison-Wesley, New York, 1991.

2. Shani, David and Sujana Chalasani: Exploiting niches using relationship marketing, Journal of Consumer Marketing, 9(3), pp.33-42, 1992.

3. Kotler, Philip: A generic concept of marketing, Journal of Marketing, 36 (2), pp.46-54, 1972.

4. Webster, Frederick E. Jr: The changing role of min the corporation, Journal of Marketing, 56 (October), pp.1-17, 1992.

5. Archana, B: Retailers Marketing Strategies and CRM, unpublished PhD Thesis, Osmania University, Hyderabad, 2007.

\section{APPENDIX:}

Table 1.1: Gender

\begin{tabular}{|c|c|c|c|c|c|c|c|c|c|c|c|c|c|}
\hline \multirow[b]{2}{*}{ Gender } & \multicolumn{13}{|c|}{ Selected Retail Stores } \\
\hline & 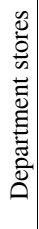 & 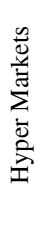 & 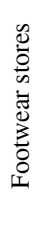 & 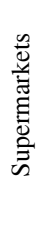 & 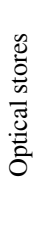 & 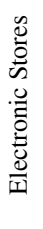 & 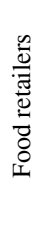 & 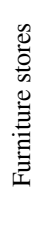 & 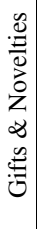 & 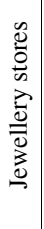 & 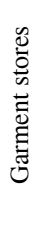 & 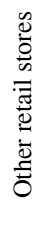 & 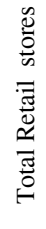 \\
\hline Male & 0 & 0 & 0 & 0 & 0 & 0 & 0 & 0 & 0 & 0 & 9 & 0 & 9 \\
\hline Female & 0 & 0 & 0 & 0 & 0 & 0 & 0 & 0 & 0 & 0 & 5 & 2 & 7 \\
\hline $\begin{array}{l}\text { Male \& } \\
\text { Female }\end{array}$ & 2 & 6 & 4 & 9 & 2 & 4 & $\begin{array}{l}1 \\
4\end{array}$ & 4 & 5 & 7 & $\begin{array}{l}1 \\
3\end{array}$ & $\begin{array}{l}1 \\
4\end{array}$ & 84 \\
\hline $\begin{array}{l}\text { Total } \\
\text { Retail } \\
\text { stores }\end{array}$ & 2 & 6 & 4 & 9 & 2 & 4 & $\begin{array}{l}1 \\
4\end{array}$ & 4 & 5 & 7 & $\begin{array}{l}2 \\
7\end{array}$ & $\begin{array}{l}1 \\
6\end{array}$ & 100 \\
\hline
\end{tabular}

Source: Questionnaire to the retailers 
Table 1.2: Age

\begin{tabular}{|c|c|c|c|c|c|c|c|c|c|c|c|c|c|}
\hline \multirow[b]{2}{*}{ Age } & \multicolumn{13}{|c|}{ Selected Retail Stores } \\
\hline & 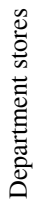 & 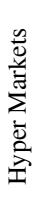 & 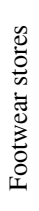 & 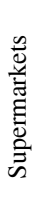 & 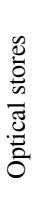 & $\begin{array}{l}0 \\
0 \\
0 \\
\text { in } \\
.0 \\
0 \\
0 \\
\frac{0}{0} \\
1\end{array}$ & 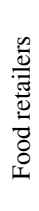 & 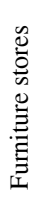 & 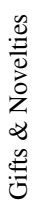 & 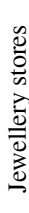 & 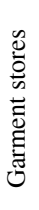 & 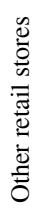 & 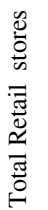 \\
\hline All age groups & 2 & 6 & 4 & 9 & 2 & 4 & 14 & 4 & 5 & 7 & 27 & 16 & 100 \\
\hline Total Retail stores & 2 & 6 & 4 & 9 & 2 & 4 & 14 & 4 & 5 & 7 & 27 & 16 & 100 \\
\hline
\end{tabular}

Table 1.3: Socio-Economic Status

\begin{tabular}{|c|c|c|c|c|c|c|c|c|c|c|c|c|c|}
\hline \multirow[b]{2}{*}{$\begin{array}{l}\text { Socio-Economic } \\
\text { Levels }\end{array}$} & \multicolumn{13}{|c|}{ Selected Retail Stores } \\
\hline & 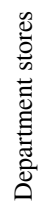 & 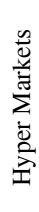 & 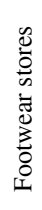 & 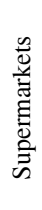 & 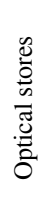 & 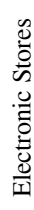 & 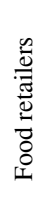 & 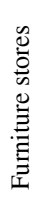 & 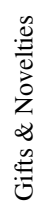 & 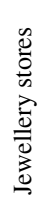 & 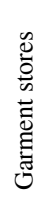 & 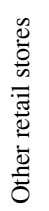 & 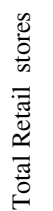 \\
\hline Very rich & 0 & 0 & 2 & 0 & 0 & 0 & 1 & 0 & 0 & 1 & 3 & 0 & 7 \\
\hline Rich & 2 & 2 & 1 & 8 & 0 & 3 & 2 & 0 & 0 & 0 & 3 & 5 & 26 \\
\hline Upper Middle & 0 & 3 & 0 & 0 & 0 & 1 & 5 & 2 & 2 & 1 & 10 & 6 & 30 \\
\hline Middle & 0 & 1 & 1 & 1 & 2 & 0 & 6 & 2 & 3 & 5 & 11 & 5 & 37 \\
\hline $\begin{array}{l}\text { Total Retail } \\
\text { stores }\end{array}$ & 2 & 6 & 4 & 9 & 2 & 4 & 14 & 4 & 5 & 7 & 27 & 16 & 100 \\
\hline
\end{tabular}

Source: Ouestionnaire to the retailers

Table 2.1: Brands sold

\begin{tabular}{|c|c|c|c|c|c|c|c|c|c|c|c|c|c|}
\hline \multirow[b]{2}{*}{ Brands } & \multicolumn{13}{|c|}{ Selected Retail Stores } \\
\hline & $\begin{array}{l}0 \\
0 \\
0 \\
0 \\
0 \\
0 \\
0 \\
000 \\
0 \\
0\end{array}$ & 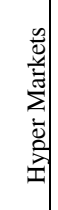 & 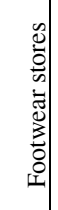 & 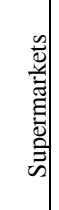 & $\begin{array}{l}0 \\
0 \\
0 \\
0 \\
0 \\
0 \\
0 \\
0\end{array}$ & 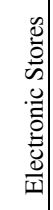 & 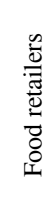 & 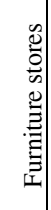 & 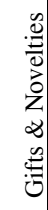 & $\begin{array}{l}0 \\
0 \\
0 \\
0 \\
7 \\
0 \\
0 \\
0 \\
0 \\
0 \\
0\end{array}$ & 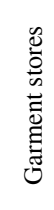 & 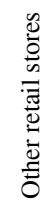 & 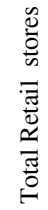 \\
\hline National & 0 & 4 & 2 & 3 & 2 & 3 & 14 & 4 & 5 & 7 & 23 & 14 & 81 \\
\hline International & 2 & 0 & 2 & 0 & 0 & 1 & 0 & 0 & 0 & 0 & 4 & 2 & 11 \\
\hline Store & 0 & 2 & 0 & 6 & 0 & 0 & 0 & 0 & 0 & 0 & 0 & 0 & 8 \\
\hline Total Retail stores & 2 & 6 & 4 & 9 & 2 & 4 & 14 & 4 & 5 & 7 & 27 & 16 & 100 \\
\hline
\end{tabular}

Source: Questionnaire to the retailers 
Table2.2: Pricing strategies

\begin{tabular}{|c|c|c|c|c|c|c|c|c|c|c|c|c|c|}
\hline \multirow[b]{2}{*}{$\begin{array}{c}\text { Pricing } \\
\text { strategies }\end{array}$} & \multicolumn{13}{|c|}{ Selected Retail Stores } \\
\hline & 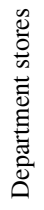 & 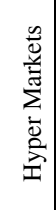 & 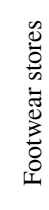 & 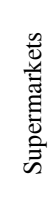 & 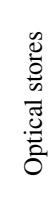 & 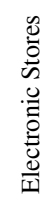 & 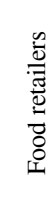 & 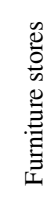 & $\begin{array}{l}\mathscr{0} \\
\stackrel{0}{0} \\
0 \\
z \\
\infty \\
\infty \\
0 \\
0\end{array}$ & $\begin{array}{l}0 \\
0 \\
0 \\
0 \\
0 \\
0\end{array}$ & 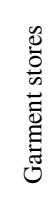 & 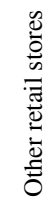 & 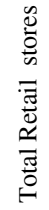 \\
\hline High & 2 & 3 & 0 & 1 & 0 & 0 & 0 & 0 & 0 & 0 & 2 & 2 & 10 \\
\hline Low & 0 & 1 & 0 & 3 & 2 & 4 & 4 & 3 & 3 & 1 & 8 & 8 & 37 \\
\hline Odd & 0 & 0 & 1 & 0 & 0 & 0 & 0 & 0 & 0 & 0 & 0 & 0 & 1 \\
\hline MRP & 0 & 2 & 3 & 4 & 0 & 0 & 10 & 1 & 2 & 6 & 17 & 6 & 51 \\
\hline EDLP & 0 & 0 & 0 & 1 & 0 & 0 & 0 & 0 & 0 & 0 & 0 & 0 & 1 \\
\hline $\begin{array}{l}\text { Total Retail } \\
\text { stores }\end{array}$ & 2 & 6 & 4 & 9 & 2 & 4 & 14 & 4 & 5 & 7 & 27 & 16 & 100 \\
\hline
\end{tabular}

Source: Questionnaire to the retailers

Table 2.3: Location criteria

\begin{tabular}{|c|c|c|c|c|c|c|c|c|c|c|c|c|c|}
\hline \multirow[b]{2}{*}{ Location criteria } & \multicolumn{13}{|c|}{ Selected Retail Stores } \\
\hline & 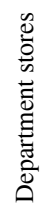 & 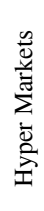 & 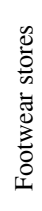 & 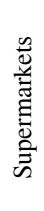 & 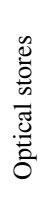 & 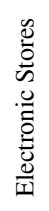 & $\begin{array}{l}\frac{0}{0} \\
\frac{0}{\pi} \\
0 \\
0 \\
0 \\
0 \\
0\end{array}$ & 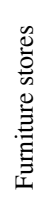 & 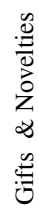 & $\begin{array}{l}0 \\
0 \\
0 \\
0 \\
0 \\
0 \\
0 \\
0 \\
0 \\
0 \\
0\end{array}$ & 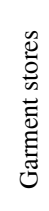 & 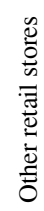 & 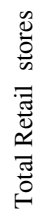 \\
\hline $\begin{array}{l}\text { Visitor group } \\
\text { attraction }\end{array}$ & 2 & 6 & 4 & 9 & 2 & 4 & 14 & 4 & 5 & 7 & 27 & 16 & \\
\hline $\begin{array}{l}\text { Large local } \\
\text { customers }\end{array}$ & 0 & 2 & 2 & 7 & 1 & 0 & 0 & 0 & 0 & 0 & 0 & 0 & \\
\hline Security & 2 & 6 & 4 & 9 & 2 & 4 & 14 & 4 & 5 & 7 & 27 & 16 & \\
\hline $\begin{array}{l}\text { Source of } \\
\text { entertainment }\end{array}$ & 2 & 2 & 1 & 1 & 0 & 1 & 5 & 0 & 0 & 2 & 5 & 4 & \\
\hline $\begin{array}{l}\text { Well connected } \\
\text { transport }\end{array}$ & 2 & 6 & 4 & 9 & 2 & 4 & 14 & 4 & 5 & 7 & 27 & 16 & \\
\hline $\begin{array}{l}\text { Planned shopping } \\
\text { area }\end{array}$ & 2 & 6 & 4 & 9 & 2 & 4 & 14 & 4 & 5 & 7 & 27 & 16 & \\
\hline Parking space & 2 & 3 & 1 & 2 & 0 & 4 & 1 & 0 & 0 & 0 & 4 & 2 & \\
\hline Occupancy costs & 2 & 6 & 4 & 9 & 2 & 4 & 14 & 4 & 5 & 7 & 27 & 16 & \\
\hline Landlord control & 2 & 6 & 4 & 9 & 2 & 4 & 14 & 4 & 5 & 7 & 27 & 16 & \\
\hline $\begin{array}{l}\text { Competition } \\
\text { factors }\end{array}$ & 2 & 6 & 3 & 8 & 1 & 2 & 2 & 2 & 1 & 5 & 10 & 8 & \\
\hline Tax incentives & 2 & 6 & 4 & 9 & 2 & 4 & 14 & 4 & 5 & 7 & 27 & 16 & \\
\hline $\begin{array}{l}\text { Protection from } \\
\text { weather }\end{array}$ & 0 & 0 & 0 & 2 & 0 & 0 & 1 & 0 & 0 & 0 & 0 & 0 & \\
\hline Total Retail stores & 2 & 6 & 4 & 9 & 2 & 4 & 14 & 4 & 5 & 7 & 27 & 16 & 100 \\
\hline
\end{tabular}


Table 3.1: Retail Practices

\begin{tabular}{|c|c|c|c|c|c|c|c|c|c|c|c|c|c|c|}
\hline \multirow{2}{*}{\multicolumn{2}{|c|}{ Retail Practices }} & \multicolumn{13}{|c|}{ Selected Retail Stores } \\
\hline & & \multirow{2}{*}{ 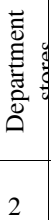 } & \multirow{2}{*}{$\begin{array}{l}\dot{\Phi} \\
\text { 空 } \\
6\end{array}$} & \multirow{2}{*}{ 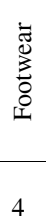 } & \multirow{2}{*}{ 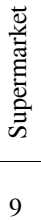 } & \multirow{2}{*}{ 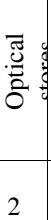 } & \multirow{2}{*}{ 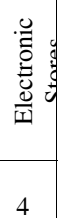 } & \multirow{2}{*}{ 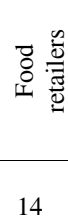 } & \multirow{2}{*}{ 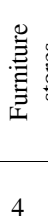 } & \multirow{2}{*}{$\begin{array}{l}\infty \\
0 \\
0 \\
0 \\
0\end{array}$} & \multirow{2}{*}{ 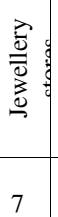 } & \multirow{2}{*}{ 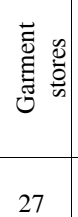 } & \multirow{2}{*}{ 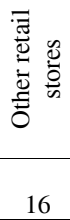 } & \multirow{2}{*}{ 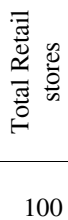 } \\
\hline $\begin{array}{l}\text { Customer } \\
\text { service }\end{array}$ & Usually & & & & & & & & & & & & & \\
\hline \multirow{3}{*}{$\begin{array}{l}\text { Customer } \\
\text { incentives }\end{array}$} & Usually & 0 & 0 & 0 & 7 & 1 & 0 & 2 & 0 & 0 & 0 & 2 & 0 & 12 \\
\hline & Sometimes & 2 & 3 & 2 & 2 & 1 & 3 & 8 & 3 & 3 & 6 & 17 & 12 & 62 \\
\hline & Rarely & 0 & 3 & 2 & 0 & 0 & 1 & 4 & 1 & 2 & 1 & 8 & 4 & 26 \\
\hline \multirow{2}{*}{$\begin{array}{l}\text { Information } \\
\text { from } \\
\text { customers }\end{array}$} & Usually & 2 & 4 & 4 & 4 & 2 & 2 & 12 & 2 & 2 & 5 & 21 & 14 & 74 \\
\hline & Sometimes & 0 & 2 & 0 & 5 & 0 & 2 & 2 & 2 & 3 & 2 & 6 & 2 & 26 \\
\hline \multirow{2}{*}{$\begin{array}{c}\text { Receive } \\
\text { complaints }\end{array}$} & Usually & 2 & 5 & 0 & 8 & 2 & 2 & 12 & 4 & 5 & 7 & 24 & 14 & 85 \\
\hline & Sometimes & 0 & 1 & 3 & 1 & 0 & 2 & 2 & 1 & 0 & 0 & 3 & 2 & 15 \\
\hline \multirow{2}{*}{$\begin{array}{l}\text { Attending } \\
\text { to customer } \\
\text { complaints }\end{array}$} & Usually & 2 & 6 & 4 & 9 & 2 & 3 & 12 & 4 & 5 & 7 & 27 & 14 & 95 \\
\hline & Sometimes & 0 & 0 & 0 & 0 & 0 & 1 & 2 & 0 & 0 & 0 & 0 & 2 & 5 \\
\hline \multirow{3}{*}{$\begin{array}{c}\text { Interaction } \\
\text { with } \\
\text { customer }\end{array}$} & Usually & 2 & 1 & 0 & 0 & 0 & 0 & 2 & 0 & 0 & 0 & 0 & 1 & 6 \\
\hline & Sometimes & 0 & 5 & 4 & 8 & 1 & 4 & 11 & 4 & 5 & 7 & 26 & 15 & 90 \\
\hline & Rarely & 0 & 0 & 0 & 1 & 1 & 0 & 1 & 0 & 0 & 0 & 1 & 0 & 4 \\
\hline \multirow{3}{*}{$\begin{array}{c}\text { Invite feed } \\
\text { back on } \\
\text { performanc } \\
\mathrm{e}\end{array}$} & Usually & 2 & 1 & 1 & 0 & 0 & 0 & 1 & 0 & 0 & 0 & 0 & 0 & 5 \\
\hline & Sometimes & 0 & 1 & 1 & 4 & 0 & 2 & 4 & 2 & 2 & 5 & 13 & 6 & 40 \\
\hline & Rarely & 0 & 4 & 2 & 5 & 2 & 2 & 9 & 2 & 3 & 2 & 14 & 10 & 55 \\
\hline \multirow{3}{*}{$\begin{array}{c}\text { Understand } \\
\text { customer } \\
\text { needs }\end{array}$} & Usually & 2 & 5 & 4 & 5 & 2 & 4 & 12 & 3 & 5 & 4 & 25 & 14 & 85 \\
\hline & Sometimes & 0 & 1 & 0 & 3 & 0 & 0 & 2 & 1 & 0 & 3 & 2 & 1 & 13 \\
\hline & Rarely & 0 & 0 & 0 & 1 & 0 & 0 & 0 & 0 & 0 & 0 & 0 & 1 & 2 \\
\hline \multirow{2}{*}{$\begin{array}{c}\text { Resolve } \\
\text { customer } \\
\text { complaints }\end{array}$} & Usually & 2 & 6 & 4 & 8 & 2 & 3 & 14 & 0 & 5 & 7 & 25 & 16 & 92 \\
\hline & Sometimes & 0 & 0 & 0 & 1 & 0 & 1 & 0 & 4 & 0 & 0 & 2 & 0 & 8 \\
\hline \multirow{3}{*}{$\begin{array}{c}\text { Customer } \\
\text { contribute } \\
\text { innovative } \\
\text { ideas }\end{array}$} & Usually & 2 & 1 & 1 & 0 & 0 & 0 & 1 & 0 & 4 & 0 & 2 & 1 & 12 \\
\hline & sometimes & 0 & 4 & 3 & 9 & 2 & 4 & 11 & 0 & 1 & 6 & 24 & 15 & 79 \\
\hline & Rarely & 0 & 1 & 0 & 0 & 0 & 0 & 2 & 4 & 0 & 1 & 1 & 0 & 9 \\
\hline \multirow{3}{*}{$\begin{array}{c}\text { Encourage } \\
\text { customers } \\
\text { to use help } \\
\text { lines }\end{array}$} & Usually & 2 & 1 & 2 & 5 & 2 & 1 & 4 & 0 & 0 & 0 & 6 & 4 & 27 \\
\hline & Sometimes & 0 & 5 & 2 & 4 & 0 & 3 & 8 & 3 & 4 & 2 & 17 & 10 & 58 \\
\hline & Rarely & 0 & 0 & 0 & 0 & 0 & 0 & 2 & 1 & 1 & 5 & 4 & 2 & 15 \\
\hline & Usually & 2 & 0 & 0 & 1 & 0 & 0 & 0 & 0 & 0 & 1 & 1 & 1 & 6 \\
\hline customers & Sometimes & 0 & 1 & 0 & 4 & 0 & 0 & 1 & 0 & 0 & 1 & 3 & 1 & 11 \\
\hline & Rarely & 0 & 5 & 4 & 4 & 2 & 4 & 13 & 4 & 5 & 5 & 23 & 14 & 83 \\
\hline $\begin{array}{l}\text { Total Retail } \\
\text { stores }\end{array}$ & & 2 & 6 & 4 & 9 & 2 & 4 & 14 & 4 & 5 & 7 & 27 & 16 & 100 \\
\hline
\end{tabular}

Table 3.2: Score for the retail practices

\begin{tabular}{|l|c|c|c|c|c|}
\hline \multicolumn{1}{|c|}{$\begin{array}{c}\text { Selected Retail } \\
\text { Stores }\end{array}$} & Mean & $\mathrm{N}$ & Std. Deviation & $\begin{array}{c}\text { Std. Error of } \\
\text { Mean }\end{array}$ & Median \\
\hline Department stores & 61.00 & 2 & .000 & .000 & 61.00 \\
\hline Hyper Markets & 48.67 & 6 & 3.445 & 1.406 & 47.00 \\
\hline Footwear stores & 51.00 & 4 & 3.651 & 1.826 & 51.00 \\
\hline Supermarkets & 49.44 & 9 & 2.603 & .868 & 51.00 \\
\hline Optical stores & 49.00 & 2 & 2.828 & 2.000 & 49.00 \\
\hline Electronic Stores & 47.00 & 4 & 2.828 & 1.414 & 46.00 \\
\hline Food retailers & 48.86 & 14 & 3.880 & 1.037 & 49.00 \\
\hline Furniture stores & 47.50 & 4 & 3.416 & 1.708 & 48.00 \\
\hline Gifts \& Novelties & 47.80 & 5 & 2.683 & 1.200 & 49.00 \\
\hline Jewellery stores & 49.29 & 7 & 2.138 & .808 & 49.00 \\
\hline Garment stores & 49.00 & 27 & 3.843 & .740 & 49.00 \\
\hline Other retail stores & 49.00 & 16 & 3.795 & .949 & 49.00 \\
\hline Total & 49.14 & 100 & 3.774 & .377 & 49.00 \\
\hline
\end{tabular}

